\title{
Internet of Things (IoT): A Revolutionary Approach towards Healthcare Surveillance
}

\author{
Vidya N L*, Ravi P, Nithin Kumar \\ Department of Computer Science and Engineering, Vidyavardhaka College of Engineering \\ Mysuru, Karnataka, India.
}

DOI: https://doi.org/10.21467/proceedings.1.43

* Corresponding author email: Vidyanlgowda1995@gmail.com

\begin{abstract}
The development of, Internet of things (IOT) makes all objects interconnected and it has been recognized as the technical revolution. One of the application is in heath care to monitor the patient health status, internet of things makes medical equipments more efficient by allowing real time monitoring of patient health, in which acquire data of patient's and reduce the human errors. In internet of things patient's parameters get transmitted through medical devices via a gateway, where it is stored and analyzed. The significant challenges in the implementation of internet of things for healthcare applications are monitoring all patients from various places. Thus, internet of things in the medical field brings out the solutions for effective patient monitoring at reduced cost and also reduces the trade- off between patient outcome and disease management. The paper analyzes the different techniques used to monitor the patients.

Keywords - IOT, Arduino Board, Sensors, Patients, health care, wireless healthcare monitoring.
\end{abstract}

\section{Introduction}

Internet-of Things (IoT)-driven health and wellness monitoring systems enable remote and continuous monitoring of individuals, with applications in chronic conditions such as obesity, hypertension, diabetes, hyperlipidemia, heart failure, asthma, depression, elderly care support, preventive care and wellness. The IoT paradigms can play a significant role in improving the health and wellness of subjects by increasing the availability and quality of care, and dramatically lowering the treatment costs and frequent travel. The IoT-driven healthcare system employs networked biosensors to simultaneously collect multiple physiological signals and wireless connectivity to share/transmit gathered signals directly to the cloud diagnostic server and the caregivers for further analysis and clinical review. Further, the IoT-enabled remote monitoring applications can significantly reduce travel, cost and time in long-term monitoring applications.

Internet of Things (IoT), gather and share information directly from patients and it also make possible to collect, record and analyze new Data Stream faster and more accurately. As the technology for collecting, analyzing and transmitting data in the IoT continues to mature, with

(C) 2018 Copyright held by the author(s). Published by AIJR Publisher in Proceedings of the $3^{\text {rd }}$ National Conference on Image Processing, Computing, Communication, Networking and Data Analytics (NCICCNDA 2018), April 28, 2018.

This is an open access article under Creative Commons Attribution-NonCommercial 4.0 International (CC BY-NC 4.0)

license, which permits any non-commercial use, distribution, adaptation, and reproduction in any medium, as long as the original work is properly cited. ISBN: 978-81-936820-0-5 
Internet of Things (IoT): A Revolutionary Approach towards Healthcare Surveillance

the help of sensors, actuators, and computing devices. This provides data communication capabilities. These are linked to networks for data transportation. This connected healthcare environment promotes the quick flow of information and enables easy access to diseases such as hypertension, diabetics and cardiac diseases which needs continuous monitoring. This Internet of Things (IoT) is increasingly recognized by the researchers and analysts as one of the most sophisticated technologies for health monitoring and it is safety for people and it also tackled by all.

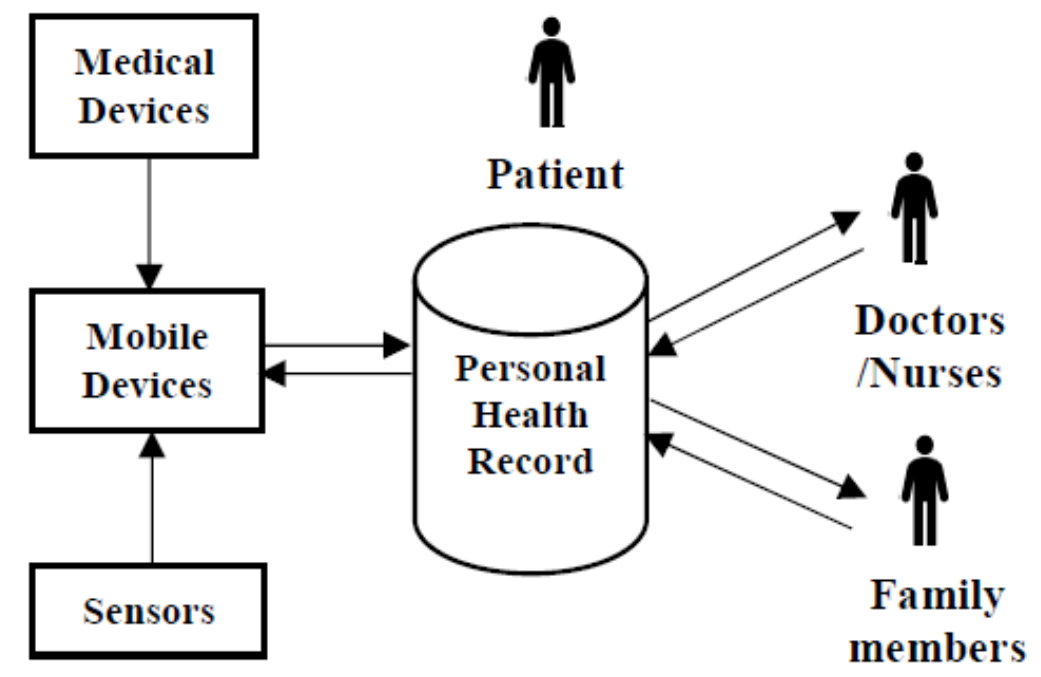

Figure 1: Structure of Io'T in healthcare

The ability of the devices to gather data on their own removes the limitations of human intervention and it reveals the data-automatically and send it to the doctor whenever they needed. The automation reduces the risk of error. This type of solution employs sensors to collect comprehensive physiological information and uses gateways and the cloud to analyze and store the information and then send the analyzed data wirelessly to caregivers for further analysis and review. It replaces the process of having a health professional come by at regular intervals to check the patient's vital signs, instead providing a continuous automated flow of information. In this way, it simultaneously improves the quality of care through constant attention and lowers the cost of care by eliminating the need for a caregiver to actively enhance in data collection and analysis. Powerful wireless solutions connected through the IoT are now making it possible for monitoring the patients. These solutions can be used to securely capture patient health data from a variety of sensors, apply complex algorithms to analyze the data and then share it through wireless network, for medical professionals who can give appropriate health recommendations for the patients.

Furthermore, the Io'T is anticipated to provide advanced connectivity of devices, systems and services that can go farther than machine to machine (M2M) communications as well as embraces various types of protocols, domains and applications. More specifically, with all 
these capabilities, the IoT will enable a new way of organizing healthcare services as well as in developing medical devices. Figure 1 illustrates the general IoT structure in healthcare. Remote health monitoring is developing increasingly day by day with the help of Internet of Things (IoT). The aim of a health monitoring system is to send patients (i.e. user's) health related information through sensor readings to a remote server (or cloud) where the remote server is accessible by clinicians, doctors, researchers, hospitals, patients etc. anywhere around the world as a characteristic of Internet of Things (IoT).

An Expert System (ES) and/or a Decision Support System (DSS) will further analyse the sensor readings through the IoT and provide a real-time feedback to the patient/user immediately. A patient/user will have the possibility to access to see/compare the current health measurement with previous measurements and same time they can share the health record with their healthcare provider. An example of such self-serve health monitoring system is presented in Figure 2 as an overview of the system.

Rest of the paper is prepared as follows: Section II gives details about the recent related work, Section III illustrates the advantages of iot healthcare, in the Section IV discussed about challenges on iot healthcare, and paper is concluded in Section V.

\section{Related work}

The respiratory rate monitor is one of the important vital signs which are useful for patients who admitted in Intensive Care Unit (ICU). The respiratory rate is calculated by using LM35 temperature sensor and monitored the patient's respiration continuously based on voltage value of inhaled and exhaled air. NRF24101 is used to transmit the sensor data from home to medical centre. Then the data are published in a web server using Ethernet to know the patient's status which is useful for doctor. And also the data is displayed in a Liquid Crystal Display (LCD). Once the threshold is reached, the alarm is generated and also a message generated in a webpage. Thereby doctor or medical professionals know the patient's conditions without delay. By using data mining techniques, people may know their condition without medical professionals help. [1]

This paper monitoring patient's body temperature and heart beat using arduino board. Health of the patients are monitored using internet o things (IoT) and enables the doctor to monitor their patients outside the clinic and also apart their consulting hours. Connected health care devices utilize resources to provide an improved quality of care, leading to better clinical outcomes. Measureable benefits of connected medical devices include reduces clinic visits, including reduction in bed days of care and length o stays in hospitals. Using Internet of Things (IoT), patient conditions are obtained and stored for further analysis. In this project the heart rate and blood pressure of patient are monitored. [2]

This paper proposes a novel signal quality aware IoT enabled ECG telemetry system for continuous cardiac health monitoring applications. The proposed quality-aware ECG

Proceedings of the $3^{\text {rd }}$ National Conference on Image Processing, Computing, Communication, Networking and Data Analytics (NCICCNDA 2018) 
Internet of Things (IoT): A Revolutionary Approach towards Healthcare Surveillance

monitoring system consists of three modules: ECG signal sensing module; automated signal quality assessment module; and signal-quality aware ECG analysis and transmission module. The main objectives of this paper are: design and development of a light-weight ECG signal quality assessment method for automatically classifying the acquired ECG signal into acceptable or unacceptable class and real-time implementation of proposed IoT-enabled ECG monitoring framework using ECG sensors, Arduino, Android phone, Bluetooth and cloud server. The proposed framework is tested and validated using the ECG signals taken from the MIT-BIH arrhythmia and Physio net Challenge databases and the real-time recorded ECG signals under different physical activities. Experimental results show that the proposed SQA method achieves promising results in identifying the unacceptable quality of ECG signals and outperforms existing methods based on the morphological and RR interval features and machine learning approaches. The study further shows that the transmission of acceptable quality of ECG signals can significantly improve the battery lifetime of IoT-enabled devices. [3]

This paper related to an android phone controlled wheel chair along with an alternative use of manual joystick. The main objective is to felicitate and increase the movement of people who are handicapped and the ones who are not able to move freely. Therefore, a design of wheelchair which will be an asset for medical department and to make it more advance in existing technology, and equipped prototype with health monitoring system involving real time measurements of body temperature, heart-rate and humidity. This will eventually decrease chances of miss-happening and allows the victim to live a freer life. [4] In this paper, they have monitored the inpatient and outpatient vital signs using Healthcare sensor and wireless technologies. They have trained a model and the model is learned by supervised learning. Meta data is also maintained for finding the health condition of patients. The model returned the status for the given vital signs, the status will be either healthy or not. [5] In this paper, they propose a new method for ECG monitoring based on Cypress Wireless Internet Connectivity for Embedded Devices (WICED) Internet of Things (IoT) platform. ECG data are gathered using a wearable monitoring node and are transmitted directly to the IoT cloud using Wi-Fi. Internet of Things utilizes open source protocols like CoAP/HTTP, MQTT, TLS/TCP, DTLS/UDP and OMALWM2M for data communication and device management. In this work they designed and implemented an ECG monitoring system based on cutting-edge cypress WICED Io'T technology. The architecture of the Io'T based ECG monitoring system was presented at the beginning. Various ECG sensing networks including Wi-Fi, Bluetooth, Zigbee and BLE were introduced and compared. Based on the proposed architecture, an IoTbased ECG monitoring system was implemented. Through a wearable monitoring node with three electrodes, real-time ECG signals can be collected with satisfactory accuracy. The gathered data were transmitted to the IoT cloud using Wi-Fi, which supports high data rates and wide coverage areas. [6]

ISBN: 978-81-936820-0-5 
This paper presents a case study on a Health-IoT system where an intelligent healthcare service is developed to monitor vital signs in daily life. Here, a generic Health-IoT framework with a Clinical Decision Support System (CDSS) is presented. The generic framework is mainly focused on the supporting sensors, communication media, secure and safe data communication, cloud-based storage, and remote accesses of the data. The CDSS is used to provide a personalized report on person's health condition based on daily basis observation on vital signs. [7] This presents five IoT technologies that are essential in the deployment of successful IoT-based products and services and discusses three IoT categories for enterprise applications used to enhance customer value. In addition, it examines the net present value method and the real option approach widely used in the justification of technology projects and illustrates how the real option approach can be applied for Io'T investment. [8]

\section{Application of IOT Healthcare}

The Internet of Things (IoT) has been making serious impact on every industry, and wherever this "technology" swept by, you can feel the Midas touch. After the "discovery" of Internet, IoT has been creating waves that not a single business in the world can deny or resist. Those who do will be long left behind as the competition is going to become stronger and tenacious. The applications of Io'T in the healthcare industry are numerous. Here are six of them:

\subsection{Real Time Location Services:}

Through IoT, doctors can use real time location services and track the devices used for treating patients. Medical staff may sometimes keep the devices in out-of-sight areas which makes them difficult to find when another medical staff comes on the scene. Medical apparatus and devices like wheelchairs, scales, defibrillators, nebulizers, pumps or monitoring equipment can be tagged with sensors and located easily with IoT. Apart from real time location services, there are IoT devices that help in environmental monitoring as well (checking the refrigerator temperature, for example).

\subsection{Predicting the Arrival of Patients in PACU:}

With the intervention of Internet of Things, clinicians can predict the arrival of patients who are recuperating in the Post-Anesthesia Care Unit (PACU). They can also monitor the status of patients in real time.

\subsection{Hand Hygiene Compliance}

There are hand hygiene monitoring systems that would detect the degree of cleanliness in a healthcare worker. According to the Centre for Disease Control and Prevention in the United States, about one patient out of every 20 gets infections from lack of proper hand hygiene in hospitals. Numerous patients lose their lives as result of hospital acquired infections. The interactions in the hand hygiene monitoring systems are done in real time and if a clinician comes near a patient's bed without washing his hands, the device would start buzzing. And

Proceedings of the $3^{\text {rd }}$ National Conference on Image Processing, Computing, Communication, Networking and Data Analytics (NCICCNDA 2018) 
Internet of Things (IoT): A Revolutionary Approach towards Healthcare Surveillance

that's not all. The information about the healthcare worker, his ID, time and location will all be fed into a database and this information would be forwarded to the concerned authorities.

\subsection{Tighten Budgets and Improve Patient Journey:}

The healthcare industry has to keep a watchful eye on the budget and at the same time have updated infrastructure to provide better patient experiences. Thanks to the seamless connection between devices that IoT has made possible, it is now possible for the medical staff to access patient information from the cloud as long as they are stored in there.

The goal is to provide quality medical care to patients and by spending a small amount on IT infrastructure, hospitals can provide good care to patients at affordable rates. IoT aims to provide better patient journey by:

$>$ Room lighting through personal control

$>$ Communicate to family and friends through email services

$>$ Immediate attention to patient needs.

\subsection{Remote Monitoring:}

Remote health monitoring is an important application of Internet of Things. Through monitoring, you can give adequate healthcare to people who are in dire need of help. Every day, lots of people die because they do not get timely and prompt medical attention. With IoT, devices fitted with sensors notify the concerned healthcare providers when there is any change in the vital functions of a person. These devices would be capable of applying complex algorithms and analyzing them so the patient receives proper attention and medical care. The collected patient information would be stored in cloud. Through remote monitoring, patients can significantly reduce the length of hospital stay and perhaps, even hospital re-admission.

\subsection{Focus on the Research Side of Healthcare:}

Protein research and composition analysis benefits from Internet of Things. Through IoT, researchers are able to analyse the accuracy of the equipment, and it rewards them by shortening their workflow through quantitative and reproducible analysis of proteins. When an infinite array of devices is connected, the healthcare industry is able to provide scalable solutions to its patients. A number of healthcare apps providing cutting-edge personalized solutions are released to them. Here are a few of them:

$>$ Medication Dispensing Device by Philips - so patients will not miss a dose anymore; perfect for elderly patients.

$>$ Niox Mino by Aerocrine - for routine measurements of Intric Oxide in a patient's breath.

$>$ UroSense by Future Path Medical - for catheterized patients to check their core body temperature and urine output.

$>$ GPS SmartSole - this is a shoe-tracking wearable device for dementia patients who have the habit of forgetting things.

ISBN: 978-81-936820-0-5 
Vidya et al., NCICCNDA 2018, AIJR Proceedings 1, pp.257-264, 2018

\section{CHALLENGES OF IOT HELATHCARE}

The following are five challenges of IoT in healthcare that put it at risk of failure.

\subsection{Lack of EHR system integration:}

While the data that is collected from Io'T devices can include a patient's vital signs, physical activity or glucose levels while at home, that information does not typically travel to an EHR system and, in most cases, is not centralized or made easily available to providers. This limits the information's value since it is not always presented to the provider in a clinical context.

\subsection{Interoperability challenges keep IoT data in different silos:}

Patients are likely to collect different sets of data when using different medical devices depending on each device's purpose and, in some cases, the ordering physician. A patient with diabetes may frequently collect glucose levels and report them back to their primary care physician while also potentially capturing data related to their asthma on a separate device, which may be going to their asthma and allergy care provider.

\subsection{IoT data alone may not be as meaningful if it is not within the context of a full health record:}

However, many providers support the collection of meaningful patient data between visits, but this data is only valuable if it can be incorporated and viewed within the context of a full patient chart and timeline. There are still many cases where the data collected from wearables and other medical devices stays locked in the IoT vendor repository or apps, but for a physician, that data may not provide any help unless it is visible within the context of the patient's full record.

\subsection{Data security causes concerns in the implementation of IoT in healthcare:}

From the time that the data is collected at the device level to the point that it is transmitted over to its final destination, securing that information is critical and is required under HIPAA. But with the lack of common security standards and practices, many health IT professionals have concerns about the risks associated with IoT device tampering and data breaches.

\subsection{Constant changes in hardware and connectivity technology:}

Patients today need more than one device to capture the different health data their providers need. This can require more than one sensor that, in most cases, is used alongside a hub to which information is pushed that's designed to process the information. These hubs are not always compatible with the different sensors that are available and having a lack of common hardware or wireless connectivity standards -- such as Wi-Fi, Bluetooth or Z-Wave -- can cause patients to have extensive hardware in their homes, which can be overwhelming and costly.

Proceedings of the $3^{\text {rd }}$ National Conference on Image Processing, Computing, Communication, Networking and Data Analytics (NCICCNDA 2018) 


\section{Conclusion}

This paper briefly reviews the advance trends in smart healthcare as an IoT application that has transformed the conventional medical system. Smart healthcare systems have reduced the complexity and the complications associated with the use of IoT environment. The vital information regarding the patient's health is recorded by the deployed IoT objects leading to efficient decision making. This paper addresses the various ways in which the healthcare can be provided to the needful patient through remote monitoring, elderly care management, virtual consultancy etc. Also there is a need to provide security and privacy of the healthcare data to improve quality of life of every person associated. This survey highlights the current challenges and issues imposing threats to the researchers. Hence a standardized framework should be proposed which will reduce security risk and overcome the open issues of constrained environments, cost-effectiveness, scalability and interoperability of healthcare applications.

\section{References}

[1] A.Raji, P.Kanchana Devi, P.Golda Jeyaseeli, N.Balaganesh "Respiratory Monitoring System for Asthma Patients" 2016 Online International Conference on Green Engineering and Technologies (IC-GET).

[2] Chesti. Altaff Hussian, K. Vuha, M. Rajani, J. Madhu Vineeth, "Smart Health Care Monitoring using Internet of Things and Android" International Journal of Advanced Research in Electronics and Communication Engineering (IJARECE) Volume 6, Issue 3, March 2017

[3] Udit Satija, Barathram.Ramkumar, and M. Sabarimalai Manikandan, Real-Time Signal Quality-Aware ECG Telemetry System for IoT-Based Health Care Monitoring, IEEE, 2017.

[4] Shubham Sagar Nayak, Prateek Gupta, Upasana, Atul B. Wani, "Wheel Chair with Health Monitoring System Using IoT", International Research Journal of Engineering and Technology (IRJET) Volume: 04 Issue: 05 | May -2017.

[5] T.Saraswathi, S.Amutha, “ IoT Based Wireless Healthcare Monitoring” International Journal of Emerging Trends in Science and Technology,2016.

[6] Uttam U. Deshpande, Milan A. Kulkarni “IoT based Real Time ECG Monitoring System using Cypress WICED”, International Journal of Advanced Research in Electrical, Electronics and Instrumentation Engineering, Vol. 6, Issue 2, February 2017.

[7] Mobyen Uddin Ahmed, “An Intelligent Healthcare Service to Monitor Vital Signs in Daily Life - A Case Study on Health-IoT”, International Journal of Engineering Research and Application, march 2017.

[8] In Lee, Kyoochun Lee, “The Internet of Things (IoT): Applications, investments, and challenges for enterprises”, Published by Elsevier, 2015.

[9] Akshay Gapchup, Ankit Wani,Durvesh Gapchup, Shashank Jadhav, "Health Care Systems Using Internet of Things", International Journal of Innovative Research in Computer and Communication Engineering, Vol. 4, Issue 12, December 2016. 